\title{
Thermodynamics of attractive hard rods: a test of mean field density functional theory
}

\author{
Sascha Khakshouri and Ian J. Ford \\ Department of Physics and Astronomy, University College London, \\ Gower Street, London WC1E 6BT, United Kingdom.
}

\begin{abstract}
Mean field density functional theory (MFDFT) has been employed to calculate the free energy of a pair of attractive hard rods on a ring. The results for homogeneous and optimal inhomogeneous density profiles have been compared with the exact free energy as a test of the approach. We discuss the problems in applying MFDFT to small systems, and suggest modifications which allow a reasonably accurate treatment of this particular, rather extreme case.
\end{abstract}

\section{INTRODUCTION}

Although the gaseous state of matter is formed primarily from independently moving molecules, solid- and liquid-like molecular clusters are present as well. These are delicate structures, changing size frequently by processes of evaporation and condensation. They are of interest because they can tell us a great deal about the forces which are responsible for the cohesion of traditional bulk condensed matter. They become particularly important when the pressure exceeds the saturated vapour pressure and the gas becomes metastable. It is through the formation and growth of molecular clusters that a supersaturated gas, with a pressure higher than the saturated vapour pressure, makes its transition to the more stable condensed phase. The properties of clusters therefore fundamentally control the process of nucleation of droplets or crystals from a vapour phase $\mathrm{s}^{1,2}$.

Interest in this process has driven efforts to calculate the thermodynamic properties of small molecular clusters. The principal property of interest in droplet nucleation is the free energy of formation of a cluster from its separated constituents, but major difficulties are encountered in computing the entropic contribution to the free energy. Molecular simulation provides the most direct approach, using Monte Carlo or molecular dynamics techniques, together with thermodynamic integration or grand canonical umbrella sampling to evaluate free energy differences ${ }^{3-11}$. However, for large clusters such methods become very time-consuming, and alternatives on a coarser level of description have been developed.

The main method available in this coarse grained category is (classical) mean field density functional theory (MFDFT). This approach has been used to study molecular clusters for nearly 20 years ${ }^{12-17}$. It is relatively simple to implement and it provides useful insight into cluster thermodynamics. It incorporates important features absent from simpler descriptions, such as the capillarity model and its derivatives ${ }^{1,18-20}$. When used to calculate droplet nucleation rates, it occupies the middle ground between the classical theory of nucleation ${ }^{21}$ and theories based on molecular simulation.

In spite of this frequent usage, however, there has been relatively little work on validating MFDFT against other approaches. This is perhaps due to the lack of suitable cases in statistical physics where analytical solution is possible. Numerical work on more microscopic scales is difficult, as mentioned earlier. One of the few such studies is the recent work by Reguera et $\mathrm{al}^{22}$, where MFDFT is compared with Monte Carlo calculations for clusters of 80 argon atoms, with a discussion of the differences in density profiles and system pressures.

In this study we consider a case where the exact free energy is available for comparison with MFDFT calculations. The reference calculations are easy since we consider a small number of molecules. On the other hand, this requires us to proceed carefully, since there are features of standard MFDFT which are inconsistent when applied to systems containing small numbers of molecules. We shall explore and comment on a number of these matters.

As is suggested by its name, MFDFT is a mean field approach, whereby the (usually pairwise) molecular interactions are represented to a first approximation by an effective external field; a potential well that confines the molecules to a certain spatial region ${ }^{16}$. Such an approach might be inaccurate for small systems. This is exacerbated by a second assumption, the random phase approximation, according to which contributions to the free energy from correlations in molecular positions are ignored. Standard MFDFT also tends to overcount the pairwise interaction energy, by overestimating the two-particle distribution function. A final problem is that the confinement of the molecules in the effective potential leads to an underestimate of the system entropy: the molecules are in fact free to move anywhere within the system and not only within the range of the effective potential ${ }^{16}$ : this requires what is called the translational correction. All of these approximations are questionable, and some of them are particularly so when the number of molecules in the system is small.

We have studied a very simple system to see how these approximations affect the accuracy of free energies calculated by MFDFT, and how the difficulties might be overcome. We have actually chosen a system where the problems should be most severe: a one dimensional system containing just two interacting hard rods. There have been few other attempts to develop a MFDFT for small systems ${ }^{23}$. In the following sections we show how MFDFT calculations of the free energy of this system compare with exact values. We illustrate some of the difficulties in the standard theory, but show how modifications of the functional allow a reasonable accuracy to be achieved. These modifications should prove useful in more complicated systems as well. We evaluate the exact free energy in section 2, develop an MFDFT in section 3 and discuss results in section 4, finally reaching some conclusions in section 5 . 


\section{EXACT FREE ENERGY}

We consider two hard rods each of length $b$, and free to move on a line of length $R$, with periodic boundary conditions. The rods interact through a pair potential $\phi\left(r_{12}\right)$ where $r_{12}$ is the separation of the rod centres. The partition function is

$$
Z=\frac{1}{2} \gamma^{2} \int_{0}^{R} d r_{1} \int_{0}^{R} d r_{2} \exp \left(-U\left(r_{1}, r_{2}\right) / k T\right),
$$

where $\gamma=(2 \pi m k T)^{1 / 2} / h$ is the inverse thermal de Broglie wavelength ( $m$ is the rod mass and $h$ is Planck's constant), $k$ is Boltzmann's constant, $T$ is the temperature, and $U\left(r_{1}, r_{2}\right)$ is the potential energy of the system: $r_{1}$ and $r_{2}$ are the positions of the centres of the rods. $U\left(r_{1}, r_{2}\right)$ can be separated into an attractive potential $\phi\left(r_{1}-r_{2}\right)$, and a hard sphere repulsive potential $U_{R}\left(r_{1}-r_{2}\right)$. Due to the periodic boundary conditions, the rods interact with each other and with periodic images. We assume a form $\phi(r)=-\alpha \exp (-\lambda r)$, and for simplicity, we limit the range of this potential so that there are only two terms in the exponent, an interaction between rod 1 and rod 2, and between rod 1 and the closest periodic image of rod 2. Further contributions to the interaction energy are neglected. We consider a short range repulsive potential $U_{R}\left(r_{1}-r_{2}\right)$ taking the form of a hard sphere interaction in one dimension:

$$
U_{R}\left(r_{1}-r_{2}\right)=0 \text { when }\left|r_{1}-r_{2}\right| \geq b, \quad U_{R}\left(r_{1}-r_{2}\right)=\infty \text { when }\left|r_{1}-r_{2}\right|<b .
$$

This reduces the partition function to $Z=\gamma^{2} R I / 2$ where

$$
I=\int_{b}^{R-b} d r_{2} \exp \left(\frac{\alpha}{k T} \exp \left(-\lambda r_{2}\right)+\frac{\alpha}{k T} \exp \left(-\lambda\left(R-r_{2}\right)\right)\right) .
$$

The free energy is $F=-k T \ln Z$, but for convenience we focus on an excess free energy, obtained by subtracting the free energy of two non-interacting point particles:

$$
F_{e x}=F-F_{i d}=-k T\left(\ln \frac{\gamma^{2} R I / 2}{\gamma^{2} R^{2} / 2}\right)=k T \ln (R / I) .
$$

The integral $I$ can be calculated numerically once the parameters are specified. For convenience, we shall later on represent distances $R, r_{2}, b$ and $\lambda$ as dimensionless multiples of some length scale $R_{0}$. We also express energies $\alpha$ and $F_{e x}$ in terms of the energy scale $k T$. It is therefore simple to evaluate the excess free energy for two interacting rods as a function of the size $R$ of the system in which they reside.

\section{MEAN FIELD DENSITY FUNCTIONAL THEORY}

\section{A. The standard free energy functional}

At the core of MFDFT applied to fluids is the separation of the attractive and repulsive interactions. To make the problem tractable, the repulsive interactions are dealt with in the hard sphere (or in this one dimensional case hard rod) approximation. The attractions are regarded as a perturbation on the hard sphere system. Next, a mean field approximation is employed which introduces an external effective single particle potential $V(r)$, tailored to mimic the effect of the pairwise attractive interactions. In order to do this, the Hamiltonian of the system is written ${ }^{16}$

$$
H=K+H_{0}+H_{1}
$$

where $K$ is the kinetic energy part of the Hamiltonian, and $H_{0}$ is the Hamiltonian

$$
H_{0}=\sum_{<i j>} U_{R}\left(r_{i}-r_{j}\right)+\sum_{i=1}^{N} V\left(r_{i}\right)
$$

where $U_{R}$ is the hard sphere repulsive interaction potential. The angled brackets signify all pairs of particles. The Hamiltonian $K+H_{0}$ describes a reference system of $N$ hard spheres (or rods) in a single particle (mean field) potential $V(r) . H_{1}$ is

$$
H_{1}=\sum_{<i j>} \phi\left(r_{i}-r_{j}\right)-\sum_{i=1}^{N} V\left(r_{i}\right)
$$


where $\phi(r)$ is the attractive interaction potential. This contribution is treated as a perturbation of the reference system in order to estimate the free energy. The exponential $\exp \left(-H_{1} / k T\right)$ is expanded to first order. The mean field free energy of the system is then given by

$$
F_{M F} \approx-k T \ln \frac{\gamma^{N}}{N !} \int \prod_{i=1}^{N} d r_{i} e^{-H_{0} / k T}\left(1-H_{1} / k T\right)=F_{0}-k T \ln \left(1-\left\langle H_{1}\right\rangle_{0} / k T\right) \approx F_{0}+\left\langle H_{1}\right\rangle_{0}
$$

where $F_{0}=-k T \ln Z_{0}$ is the free energy of the reference system, with $Z_{0}=\left(\gamma^{N} \int \prod d r_{i} \exp \left(-H_{0} / k T\right) / N !\right)$. The second term is the average perturbative energy estimated using the probability distribution of states in the reference system. Spatial integrations are over the entire ring. The free energy depends on the choice made for the mean field potential $V(r)$. However, the average of $V(r)$ cancels out between the two contributions in equation (8), allowing the free energy to be rewritten as

$$
F_{M F}=F_{h}+\left\langle\sum_{<i j>} \phi\left(r_{i}-r_{j}\right)\right\rangle_{0}
$$

where $F_{h}$ is the free energy of the reference system minus the contribution due to the mean field potential, and the second term is the average of the pairwise attractive interactions in the ensemble of the reference system.

The mean field potential $V(r)$ appears neither in the full Hamiltonian in equation (5), nor explicitly in the free energy $F_{M F}$ given by equation (9). The dependence on $V(r)$ is hidden in the weighting of configurations in the $\langle\ldots\rangle_{0}$ averages and in $F_{h}$. The main purpose of $V(r)$ is to mimic the effect of the attractive interactions. This approximation is best when the perturbative contribution to equation (8) is small. This can be achieved by choosing $V(r)$ to minimise the free energy $F_{M F}$. The Bogoliubov inequality ensures that the free energy calculated in this way will not lie below the true free energy of the system with Hamiltonian $H^{24}$.

We can develop the theory now in terms of particle density profiles: a density functional approach. Both terms on the right hand side of equation (9) may be regarded as functionals of the reference system single particle distribution function or density profile $\rho(r)$, defined by

$$
\rho(r)=\left\langle\sum_{i=1}^{N} \delta\left(r_{i}-r\right)\right\rangle_{0}=\frac{N}{Z_{0}} \frac{\gamma^{N}}{N !} \int \prod_{i=1}^{N} d r_{i} \exp \left(-H_{0} / k T\right) \delta\left(r_{1}-r\right) .
$$

This is itself a functional of the mean field potential $V(r)$, so the minimisation of the free energy, now written $F_{D F T}$, may be taken to be with respect to this density profile. This was the key observation made by Barrett in his interpretation of the standard density functional approach ${ }^{16}$.

Let us now develop a density functional representation of the free energy $F_{D F T}$ for the two-rod system. The term $F_{h}$ is the intrinsic free energy of an inhomogeneous fluid of non-attractive hard rods. Percus ${ }^{25,26}$ developed an analytical functional for this, which is exact for the case when the number of particles $N$ is large. It will be shown that this treatment breaks down for the case when $N=2$. For the purposes of this study we employ the local density approximation (LDA), since this is the standard practice for developing MFDFT in higher dimensions, where exact solutions are not available.

The first step is to find an expression for the free energy of a homogeneous system of hard rods. In the notation of the preceding section, the exact free energy of two non-interacting hard rods is $F_{h}=-k T \ln \left(\gamma^{2} R(R-2 b) / 2\right)$. The homogeneous one-particle density is $\rho=2 / R$ so we can rewrite this as

$$
F_{h}=2 k T \ln \left(\frac{2}{\gamma R} \frac{1}{(2(1-2 b / R))^{1 / 2}}\right),
$$

and extract the free energy density

$$
f_{h}=F_{h} / R=k T \rho \ln \left(\frac{\rho}{\gamma} \frac{1}{\left(2(1-b \rho)^{1 / 2}\right.}\right) .
$$

The next step is to write the free energy in the local density approximation as a functional of a spatially varying, reference system one-particle density $\rho(r)$ :

$$
F_{h}=\int_{0}^{R} f_{h}(\rho(r)) d r=\int_{0}^{R} k T \rho(r) \ln \left(\frac{\rho(r)}{\gamma} \frac{1}{\left(2(1-b \rho(r))^{1 / 2}\right.}\right) .
$$

This is the intrinsic free energy of the system with Hamiltonian $H_{0}$, that is to say the contribution due to the interactions between the rods and the mean field has been subtracted. It is clearly a functional of $\rho(r)$. 
The second term in equation (9) is the average attractive energy evaluated in the probability distribution of states in $H_{0}$, which can be written in the form ${ }^{27}$

$$
\left\langle\sum_{<i j>} \phi\left(r_{i}-r_{j}\right)\right\rangle_{0}=\frac{1}{2} \int_{0}^{R} d r \int_{0}^{R} d r^{\prime} \rho^{(2)}\left(r, r^{\prime}\right) \phi\left(r-r^{\prime}\right)
$$

where $\rho^{(2)}\left(r, r^{\prime}\right)$ is the two-particle distribution function of hard rods in the reference system. The standard DFT treatment of this contribution involves a further simplification: the random phase approximation (RPA), according to which

$$
\rho^{(2)}\left(r, r^{\prime}\right) \approx \rho(r) \rho\left(r^{\prime}\right)
$$

and the final expression for $F_{D F T}$ is then

$$
F_{D F T}=\int_{0}^{R} d r k T \rho(r) \ln \left(\frac{\rho(r)}{\gamma} \frac{1}{\left(2(1-b \rho(r))^{1 / 2}\right.}\right)+\frac{1}{2} \int_{0}^{R} d r \int_{0}^{R} d r_{2}^{\prime} \rho(r) \rho\left(r^{\prime}\right) \phi\left(r-r^{\prime}\right) .
$$

The free energy is now explicitly a functional of the reference system one-particle density profile $\rho(r)$. Minimising equation (16) with respect to $\rho(r)$ is equivalent to choosing the $V(r)$ which best mimics the attractive interactions.

The optimal $V(r)$ can be reconstructed from the optimal density profile if required, though there are difficulties which we shall discuss shortly.

The minimisation is achieved by solving the Euler-Lagrange equation associated with equation (16), incorporating the normalisation constraint $\int \rho d r=2$ through a Lagrange multiplier $\Lambda$ :

$$
\mu_{h}(\rho(r))=\Lambda-\int \rho\left(r^{\prime}\right) \phi\left(r-r^{\prime}\right) d r^{\prime}
$$

where

$$
\mu_{h}(\rho(r))=\frac{d f_{h}(\rho(r))}{d \rho}=k T\left[\ln \left(\frac{\rho(r)}{\gamma(2(1-b \rho(r)))^{1 / 2}}\right)+1+\frac{b \rho(r)}{2(1-b \rho(r))}\right] .
$$

The free energy of the system is then obtained by substituting this profile into the original free energy functional. This completes our development of standard MFDFT for this problem.

At this point we can compare the intrinsic chemical potential of the hard rod fluid $\mu_{h}$ to that obtained by Percus. ${ }^{26}$

$$
\mu_{h}[\rho(r)] / k T=\ln \left[\frac{\rho(r)}{1-\int_{r}^{r+b} \rho\left(r^{\prime}\right) d r^{\prime}}\right]+\int_{r-b}^{r} \frac{\rho\left(r^{\prime}\right) d r^{\prime}}{1-\int_{r^{\prime}}^{r^{\prime}+b} \rho\left(r^{\prime \prime}\right) d r^{\prime \prime}}
$$

Setting $\rho(r)$ to a constant $\rho$, this equals

$$
\mu_{h}[\rho] / k T=\ln \left[\frac{\rho}{1-b \rho}\right]+\frac{b \rho}{1-b \rho}
$$

The difference between this and (18) with $\rho(r)=\rho$ and $\gamma=1$ arises because the Percus formula was derived in the thermodynamic limit.

Note that in calculations in nucleation theory, the grand potential $\Omega$ is often required, in which case the constant $\Lambda$ in the above equation is the fixed external chemical potential, and $\Omega=F_{D F T}-\Lambda N$ with $N$ now equal to the mean number of molecules in the system for a given chemical potential. For our closed system, the chemical potential $\Lambda$ is adjusted to ensure that the number of particles present is equal to two.

\section{B. Higher order contributions to $\rho$}

It is worth reflecting briefly on the density profile that emerges from the above procedure. It is quite natural for the resulting profile to be spatially varying. This is just a consequence of the spatial dependence of the mean field potential $V(r)$ present in the reference system Hamiltonian. On the other hand, the single-particle density profile $\rho_{H}$ in the real (not the reference) system is given by

$$
\rho_{H}(r)=\frac{N}{Z} \frac{\gamma^{N}}{N !} \int \prod_{i=1}^{N} d r_{i} \exp \left(-\left(H_{0}+H_{1}\right) / k T\right) \delta\left(r_{1}-r\right)
$$


where $Z$ is the exact partition function. Due to the translational symmetry of the Hamiltonian $H=K+H_{0}+H_{1}$, this profile should be uniform. The reference density profile $\rho(r)$ can be considered as the zeroth order approximation to $\rho_{H}(r)$ in an expansion in $H_{1}$. To check the validity of this approach, higher order contributions to $\rho_{H}$ can be computed by expanding the $\exp \left(-H_{1} / k T\right)$ factor in equation (21). For example the first order density profile is given by

$$
\rho_{1}(r)=\frac{N \int \prod_{i=1}^{N} d r_{i} \exp \left(-H_{0} / k T\right)\left(1-H_{1} / k T\right) \delta\left(r-r_{1}\right)}{\int \prod_{i=1}^{N} d r_{i} \exp \left(-H_{0} / k T\right)\left(1-H_{1} / k T\right)} .
$$

This can be calculated approximately, using the random phase approximation and the zero order density profile $\rho(r)$ :

$$
\rho_{1}(r) \approx \frac{\rho(r)\left[1-\frac{1}{2}(N-1)(N-2) N^{-2} \mathrm{I}_{1}+(N-1) N^{-1} \mathrm{I}_{2}-(N-1) N^{-1} \int d r^{\prime} \phi\left(r-r^{\prime}\right) \rho\left(r^{\prime}\right) / k T+V(r) / k T\right]}{1-\frac{1}{2}(N-1) N^{-1} I_{1}+I_{2}}
$$

where

$$
\mathrm{I}_{1}=\int d r^{\prime} d r^{\prime \prime} \phi\left(r^{\prime}-r^{\prime \prime}\right) \rho\left(r^{\prime}\right) \rho\left(r^{\prime \prime}\right) / k T
$$

and

$$
\mathrm{I}_{2}=\int d r^{\prime} V\left(r^{\prime}\right) \rho\left(r^{\prime}\right) / k T
$$

One problem here is that the mean field $V(r)$ appears explicitly in equation (23). The inversion of the zeroth order profile $\rho(r)$ to give $V(r)$ is tedious, and also incomplete, since the mean field is necessarily uncertain up to a constant. This difficulty does not occur in earlier expressions such as equation (16), where the role played by the mean field is hidden. However, we may proceed using an approximation for $V(r)$ obtained by neglecting the hard sphere repulsions, namely $V(r) \approx-k T \ln \rho(r)+C$ where $C$ is the arbitrary constant. It is possible to fix this constant by demanding that the perturbative second term in equation (8), evaluated using the approximate $V(r)$, should vanish. Once this is imposed, the first order density profile reduces to

$$
\rho_{1}(r) \approx \rho(r)\left[1-\frac{(N-1)}{N^{2}} \mathrm{I}_{1}-\frac{1}{N} \mathrm{I}_{2}-\frac{(N-1)}{N} \int d r^{\prime} \phi\left(r-r^{\prime}\right) \rho\left(r^{\prime}\right) / k T-\ln \rho\right]
$$

in which $I_{2}$ is now the integral in equation (25) with $V(r)$ replaced by $-k T \ln \rho$.

This result is still spatially varying. Indeed any expansion of equation (21) to finite order, and employing approximations to calculate the corrections, will lead to a density profile with spatial variation. The loss of translational symmetry is caused fundamentally by the need to choose an arbitrary location in space for the centre of the mean field potential well. By choosing a particular location and using the zeroth order term, we obtain an inhomogeneous reference system single-particle density profile $\rho(r)$. This is the interpretation by Barrett ${ }^{16}$. The breakage of translational symmetry, however, is associated with an undesirable error in the free energy. The MFDFT approach should be amended to take this effect into account, and we shall address this point later on.

It is also worth pointing out that the Euler-Lagrange equation (17) generates profiles corresponding to stationary values of the free energy or grand potential. Saddle point solutions are produced as well as stable global minima, and in nucleation applications, the metastable solutions are identified with critical clusters: the growth or evaporation of which are equally likely. The objection has been made that saddle point solutions are irrelevant. For given external conditions (say temperature and chemical potential) the system has a unique free energy and grand potential, and it is only the minimised free energy from the MFDFT approach that can be taken as an approximation to the free energy of an actual system. To counter this objection, it was shown by Talanquer and Oxtoby ${ }^{14}$ that the metastable solutions in an open system (fixed chemical potential) map onto stable solutions of a closed system (with fixed $N$ ), and so the use of MFDFT might thereby be justified. It is necessary, however, to make a choice of the volume for the closed system, and this may be thought to correspond to a cluster definition. The need to define a cluster in terms of a region of $N$-particle phase space is a common feature in microscopic statistical mechanical models, but in contrast to other approaches, MFDFT appears to be free of the necessity to do likewise. This is not the case, for the reasons just outlined. Furthermore, the cluster definitions available in MFDFT seem limited to the imposition of a confining volume, rather than something more physical, such as conditions involving binding energy ${ }^{28}$.

\section{Method of solution}

There is a standard iterative approach to solving the integral equation (17) for $\rho(r)$. A trial profile is inserted into the right hand side, and a new profile is generated by inverting the function $\mu_{h}(\rho)$. For a fixed $\Lambda$ this procedure leads ultimately to a free 
energy minimising profile, but if the initial profile is chosen suitably, then a metastable saddle point solution may emerge and remain little changed for many iterations.

This is not the procedure we use, largely because in practice we found that for small systems the metastable state is not readily observable. Instead we effectively update $\Lambda$ at each iteration with the intention of ensuring the desired normalisation condition $\int \rho d r=2$. We alter the Euler-Lagrange equation to

$$
\mu_{h}(\rho(r))=-k T \ln \left(\frac{1}{2} \int d r \exp \left(\left(-\mu_{h, c}-\phi_{\mathrm{eff}}\right) / k T\right)\right)-\phi_{\mathrm{eff}} .
$$

where $\phi_{\text {eff }}(r)=\int \rho\left(r^{\prime}\right) \phi\left(r-r^{\prime}\right) d r^{\prime}$ and $\mu_{h, c}(r)=\mu_{h}-k T \ln (\rho / \gamma)$. This revised equation is equivalent to that employed by Lee et $\mathrm{al}^{12}$ and Talanquer and Oxtoby ${ }^{14}$ to control the normalisation for closed systems. The first term on the left hand side of equation (27) is nothing more than the appropriate value of $\Lambda$ appearing in equation (17). The density profile generated from this procedure is then inserted into the free energy functional, equation (14), to obtain the MFDFT estimate of the free energy of the system.

\section{Modifications}

\section{Small $N$ effects}

In applying MFDFT to small systems, several crucial modifications to the standard development should not be overlooked. These modifications are necessary to take account of finite size effects that become increasingly important as the number of particles becomes small.

The first two corrections take us back to the random phase approximation (RPA) in equation (15). The correct relationship between the two-particle distribution function $\rho^{(2)}\left(r, r^{\prime}\right)$, and a product of two single particle distribution functions $\rho(r) \rho\left(r^{\prime}\right)$ is given by

$$
\rho^{(2)}\left(r, r^{\prime}\right)=g\left(r, r^{\prime}\right) \rho(r) \rho\left(r^{\prime}\right),
$$

where $g\left(r, r^{\prime}\right)$ is the pair correlation function. In the RPA this function is set to unity irrespective of the positions $r$ and $r^{\prime}$. We will make corrections to this approximation in several stages. The first correction results from consideration of the normalisation condition $^{27}$ for an $n$-particle distribution function:

$$
\int_{0}^{R} \prod_{i=1}^{n} d r_{i} \rho^{(n)}\left(\left\{r_{i}\right\}\right)=\frac{N !}{(N-n) !}
$$

For the case of $n=2$ (the two-particle distribution function) the right hand side of equation (29) is equal to $N(N-1)$. On the other hand, the one-particle distribution functions are normalised to $N$, giving $N^{2}$ for the integral of the product $\rho(r) \rho\left(r^{\prime}\right)$. Therefore the RPA approximation $g=1$ should be replaced by the more accurate form $g=(N-1) / N$, and equation (15) becomes

$$
\rho^{(2)}\left(r, r^{\prime}\right) \approx \frac{N-1}{N} \rho(r) \rho\left(r^{\prime}\right) .
$$

It is clear that this correction is only important for small $N$, but in the case of $N=2$ it introduces a substantial correction factor of $1 / 2$. We have employed this correct normalisation in the derivation of equation (23) already.

A second modification of equation (15) addresses the neglect within the RPA of spatial correlations between the two rods. Associated terms have have been ignored in the perturbative contribution to the free energy: $\frac{1}{2} \int_{0}^{R} d r \int_{0}^{R} d r^{\prime} \rho^{(2)}\left(r, r^{\prime}\right) \phi\left(r-r^{\prime}\right)$. The most severe consequence of this is that the RPA fails to remove the attractive energy due to overlapping configurations of rods i.e. when $\left|r-r^{\prime}\right|<b$. This leads to a substantial overestimation of the magnitude of the cohesive energy in the system. An approximate way to take account of this is to construct a correlation function from a pair of step functions

$$
g\left(r, r^{\prime}\right) \propto \Theta\left(\left|r-r^{\prime}\right|-b\right) \Theta\left(R-\left|r-r^{\prime}\right|-b\right),
$$

where $\Theta(z)=0$ for $z<0$ and unity otherwise. Now $g\left(r, r^{\prime}\right)$ is explicitly zero for overlapping hard rod configurations, and equation (15) is modified to

$$
\rho^{(2)}\left(r, r^{\prime}\right) \approx\left(\frac{N-1}{N}\right)\left(\frac{R}{R-2 b}\right) \rho(r) \rho\left(r^{\prime}\right) \Theta\left(\left|r-r^{\prime}\right|-b\right) \Theta\left(R-\left|r-r^{\prime}\right|-b\right),
$$

An extra factor of $R /(R-2 b)$ has been inserted to ensure that $\rho^{(2)}$ remains normalised for homogeneous profiles despite the exclusion of $2 b$ from the available volume in the system. This pair distribution function is exact for a homogeneous fluid 
of two hard rod particles, however it is an approximation when an inhomogeneous external field is present giving rise to an inhomogeneous density profile. The MFDFT free energy function is now written

$$
F_{D F T}=\int_{0}^{R} d r k T \rho(r) \ln \left(\frac{\rho(r)}{\gamma} \frac{1}{\left(2(1-b \rho(r))^{1 / 2}\right.}\right)+\frac{1}{2} \int_{0}^{R} d r \int_{0}^{R} d r_{2}^{\prime} \rho^{(2)}\left(r, r^{\prime}\right) \phi\left(r-r^{\prime}\right),
$$

employing the two-particle distribution function from equation (32).

The need for this correction is not particularly a consequence of the small size of the system, but rather the relatively low magnitude of the total cohesive energy in this two-particle case. If there were more particles in more dimensions, and therefore more pairwise contributions to the system energy, the error introduced through allowing particle overlap would be less severe.

\section{Centre of mass translation}

The final correction we need to consider deals with the breakage of translational symmetry implied by MFDFT and the consequent inhomogeneous distribution of the centre of mass of the system. A dynamical mode of the system, namely the motion of the centre of mass, is incorrectly described, and this affects the free energy.

This issue has been the subject of some controversy in the literature. Talanquer and Oxtoby ${ }^{14}$ have assumed that when MFDFT is applied to a system in a closed volume, the free energy obtained includes the full translational free energy for the centre of mass of the system within that volume. Simulations by Reguera et $\mathrm{al}^{22}$, on the other hand, indicate that density profiles obtained from MFDFT correspond closely to a system modelled by Monte Carlo simulation with a fixed centre of mass. We take the view of Barrett ${ }^{16}$, that the MFDFT approach limits the translational motion of the centre of mass of the system to a certain volume around the centre of the mean field potential well. For system sizes of a few tens of particles, this is a small volume compared with the extent of the profile itself, and so this interpretation is consistent with the observations made by Reguera et $\mathrm{al}^{22}$. However, the centre of mass is not fixed: it may be regarded as tethered to the midpoint of the mean field potential, and undergoing quasi-harmonic oscillations about it.

To make this clearer, consider a one dimensional closed system of length $R$, containing $N$ particles interacting through a pair potential $U\left(r_{i}-r_{j}\right)$. With suitable periodic boundary conditions that avoid boundary effects, the probability distribution for the position of the centre of mass $\rho_{c}\left(R_{c m}\right)$ should be uniform. Now, $\rho_{c}\left(R_{c m}\right)$ is the expectation value of the operator $\hat{\rho}\left(R_{c m}\right)=\delta\left(\frac{1}{N} \sum_{i=1}^{N} r_{i}-R_{c m}\right)$, so

$$
\rho_{c}\left(R_{c m}\right)=\frac{1}{Z} \frac{\gamma^{N}}{N !} \int \prod_{i=1}^{N} d r_{i} \delta\left(\frac{1}{N} \sum_{i=1}^{N} r_{i}-R_{c m}\right) e^{-\left(H_{0}+H_{1}\right) / k T}
$$

where $Z$ is the exact partition function. Transforming to centre of mass coordinates $r_{i}^{\prime}=r_{i}-R_{c m}, \rho_{c}\left(R_{c m}\right)$ becomes

$$
\rho_{c}\left(R_{c m}\right)=\frac{1}{Z} \frac{\gamma^{N}}{N !} \int \prod_{i=1}^{N} d r_{i}^{\prime} \delta\left(\sum_{i=1}^{N} \frac{1}{N} r_{i}^{\prime}\right) e^{-\left(H_{0}+H_{1}\right) / k T}
$$

$H_{0}+H_{1}$ is unchanged by this change of coordinates since it depends only on particle separations, and with the correct boundary conditions the integration limits remain unchanged. We can therefore see that the right hand side of equation (35) does not depend the value of $R_{c m}$, resulting in a distribution $\rho_{c}$ that does not depend on position.

As we have seen, in MFDFT the aim is to find the optimal form of the effective mean field potential $V(r)$ in the Hamiltonian $H_{0}$ to mimic the effect of the attractive interactions in $H_{1}$. The density profile $\rho(r)$ is the single particle distribution function arising from the reference system described by $H_{0}$ with this form of $V(r)$. The MFDFT approximation to the distribution of the centre of mass is determined by taking the trace of the operator $\hat{\rho}\left(R_{c m}\right)$ in the reference ensemble, which we write as

$$
\rho_{c}^{0}\left(R_{c m}\right)=\frac{1}{Z_{0}} \frac{\gamma^{N}}{N !} \int \prod_{i=1}^{N} d r_{i} \delta\left(\frac{1}{N} \sum_{i=1}^{N} r_{i}-R_{c m}\right) \exp \left(-\frac{1}{k T}\left(\sum_{<i j>} U_{R}\left(r_{i}-r_{j}\right)+\sum_{i=1}^{N} V\left(r_{i}\right)\right)\right) .
$$

Converting to the centre of mass coordinates $r_{i}^{\prime}=r_{i}-R_{c m}$, we get

$$
\rho_{c}^{0}\left(R_{c m}\right)=\frac{1}{Z_{0}} \frac{\gamma^{N}}{N !} \int \prod_{i=1}^{N} d r_{i}^{\prime} \delta\left(\sum_{i=1}^{N} \frac{1}{N} r_{i}^{\prime}\right) \exp \left(-\frac{1}{k T}\left(\sum_{<i j>} U_{R}\left(r_{i}^{\prime}-r_{j}^{\prime}\right)+\sum_{i=1}^{N} V\left(r_{i}^{\prime}+R_{c m}\right)\right)\right) .
$$

The integrand now does depend upon $R_{c m}$, as a result of the spatial dependence of the mean field potential $V(r)$. Consequently the centre of mass distribution $\rho_{c}^{0}$ is spatially varying: we are more likely to find the centre of mass in some parts of the system than 
in others. This remains the case to higher order in perturbation theory, along the lines described in section III B. This unphysical result is an artefact of the mean field approximation, and will lead to an underestimation of the entropy of the system. Correcting this error is possible by changing the treatment of the centre of mass dynamical degree of freedom ${ }^{29,30}$. This replacement is slightly ad hoc and unsatisfactory, but it is the treatment we shall use here.

We begin by writing the reference partition function $Z_{0}$ as

$$
Z_{0}=\frac{1}{h^{N} N !} \int \prod_{i=1}^{N} d r_{i} d p_{i} \exp \left(-\left(K+H_{0}\right) / k T\right),
$$

where the $p_{i}$ are the particle momenta. This can be expressed with the insertion of integrations over the centre of mass position $R_{c m}$ and momentum $P_{c m}$ :

$$
\begin{aligned}
Z_{0}= & \int d R_{c m} d P_{c m} \frac{1}{h^{N} N !} \int \prod_{i=1}^{N} d r_{i} d p_{i} \delta\left(\frac{1}{N} \sum_{i=1}^{N} r_{i}-R_{c m}\right) \delta\left(\sum_{i=1}^{N} p_{i}-P_{c m}\right) \\
& \exp \left(-\frac{1}{k T}\left(K+\sum_{<i j>} U_{R}\left(r_{i}-r_{j}\right)+\sum_{i=1}^{N} V\left(r_{i}\right)\right)\right),
\end{aligned}
$$

or

$$
Z_{0}=\int d R_{c m} d P_{c m} \chi\left(R_{c m}, P_{c m}\right)
$$

which defines a distribution function $\chi$, which is related to $\rho_{c}^{0}$ through

$$
\rho_{c}^{0}\left(R_{c m}\right)=\frac{1}{Z_{0}} \int \chi\left(R_{c m}, P_{c m}\right) d P_{c m}
$$

Equation (40) may be cast instead in the form

$$
Z_{0}=\frac{1}{h} \int d P_{c m} d R_{c m} \exp \left(-H_{\text {eff }}\left(R_{c m}, P_{c m}\right) / k T\right)
$$

where $H_{\text {eff }}$ is an effective Hamiltonian controlling the dynamics of the system centre of mass. In order to describe properly the freedom of motion of the centre of mass, we should replace this Hamiltonian with that of a free particle. The corrected partition function is

$$
Z_{0}^{\prime}=Z_{0} \frac{\int d R_{c m}}{\int d R_{c m} \exp \left(-U\left(R_{c m}\right) / k T\right)}
$$

where $U\left(R_{c m}\right)$ is the effective potential in the effective Hamiltonian. The origin $R_{c m}=0$ is chosen to lie at the centre of the mean field potential and we can choose $U(0)=0$. Therefore $\rho_{c}^{0}(0)=h^{-1} \int d P_{c m} \exp \left(-H_{\mathrm{eff}}\left(0, P_{c m}\right) / k T\right) / Z_{0}=$ $1 / \int d R_{c m} \exp \left(-U\left(R_{c m}\right) / k T\right)$ and we can write

$$
\frac{Z_{0}^{\prime}}{Z_{0}}=\frac{\int d R_{c m}}{\int d R_{c m} \exp \left(-U\left(R_{c m}\right) / k T\right)}=\rho_{c}^{0}(0) R
$$

which is in agreement with similar corrections suggested in the literature ${ }^{29,30}$. The associated shift in reference free energy is given by

$$
\Delta F=-k T \ln \left(\rho_{c}^{0}(0) R\right)
$$

This free energy correction may be evaluated using the optimum single particle density profile $\rho(r)$ obtained from the EulerLagrange equation. We return to equation (36) which we write in the form

$$
\rho_{c}^{0}\left(R_{c m}\right)=\frac{1}{N !} \int \prod_{i=1}^{N} d r_{i} \delta\left(\frac{1}{N} \sum_{i=1}^{N} r_{i}-R_{c m}\right) \rho^{(N)}\left(\left\{r_{i}\right\}\right),
$$

where $\rho^{(N)}$ is the $N$-body distribution function. We can proceed in terms of the one-particle density profile $\rho(r)$ by modelling the $N$-particle distribution according to a crude random phase approximation:

$$
\rho^{(N)}\left(\left\{r_{i}\right\}\right)=\frac{N !}{N^{N}} \prod_{i=1}^{N} \rho\left(r_{i}\right),
$$


so $\rho_{c}^{0}(0)$ is given by

$$
\rho_{c}^{0}(0)=\frac{1}{N^{N}} \int \prod_{i=1}^{N} d r_{i} \rho\left(r_{i}\right) \delta\left(\frac{1}{N} \sum_{i=1}^{N} r_{i}\right),
$$

which is readily calculable. For the case of $N=2$, the result is simply

$$
\rho_{c}^{0}(0)=\frac{1}{4} \int d r_{1} \rho\left(r_{1}\right) \rho\left(-r_{1}\right)=\frac{1}{4} \int d r_{1} \rho^{2}\left(r_{1}\right)
$$

with the final form being a consequence of symmetry. A better approximation, perhaps, would be to use the more appropriate version of the RPA given in equation (32).

For general $N$, we can evaluate the integral in equation (48) by inserting $\delta(x)=(2 \pi)^{-1} \int_{-\infty}^{\infty} d w \exp (i w x)$, in which case $\rho_{c}^{0}(0)=N^{-N}(2 \pi)^{-1} \int_{-\infty}^{\infty} d w \widetilde{\rho}^{N}(w / N)$, where $\widetilde{\rho}$ is given by

$$
\widetilde{\rho}(q)=\int \rho(r) \exp (i q r) d r
$$

The translational free energy correction $\Delta F$ is therefore calculable, and this completes the improved MFDFT treatment.

\section{RESULTS}

We perform calculations for two finite rod lengths $b=0.1, b=0.01$ and also point-like particles $b=0$ in dimensionless units. For each rod length, equilibrium density profiles were found by solving equation (18) and the MFDFT free energy is calculated for a range of 'volumes' $R$. This is then compared to the MFDFT free energy for a homogeneous density distribution $\rho=2 / R$, and to the exact free energy obtained by numerical integration of the partition function in equation (1).

As mentioned earlier, to avoid complications we focus on the excess free energy $F_{e x}$, which measures the difference in free energy of the system and an ideal gas consisting of two noninteracting particles $(\alpha=0$ and $b=0)$ occupying the same volume. To illustrate the qualitative differences between the interacting case and the noninteracting case, Figure 1 shows the full free energy (in arbitrary units) versus $R$ of a system of interacting particles $(\alpha=5, b=1, \lambda=0.5)$ as well as the free energy of noninteracting particles. It can be seen that at small volumes the difference is significant, due to the short range repulsive and attractive forces, whilst for large volumes the difference becomes smaller as these contributions to the free energy become less important. We therefore expect $F_{e x}$ to become small for large $R$.

Homogeneous profiles profiles are expected to be the solutions to the Euler-Lagrange equation as $\lambda R \rightarrow 0$, since in this limit, there is minor spatial dependence in the attractive interaction, and it has the same effect as adding a constant potential to the system. It can be shown that the functional in equation (33) provides the exact free energy of the system in this limit. The error compared with the exact free energy increases with $\lambda$ and $\alpha$, as shown in Figure 2 . For this study, the parameters were adjusted to give a reasonably short range interaction, with considerable strength. The depth of the attractive potential energy well at contact (i.e. when the rod centres are separated by $b$ ) has been set to $\phi(b)=-10 k T$, and the parameter $1 / \lambda$, which determines the length scale of the attractive interaction, has been set to unity. In Figures 3-5 $F_{e x}$ is plotted against $R$ for three different rod sizes.

The MFDFT free energies, obtained by finding inhomogeneous optimal density profiles, lie closer to the exact free energy, though there remains a large discrepancy. At small volumes $(R \sim 3$ for $b=0$ and $b=0.01$, and $R \sim 5$ for $b=0.1)$ the difference in free energy associated with the homogeneous and optimal inhomogeneous density profiles becomes very small, and eventually the homogeneous solutions to equation (18) become optimal. A similar development is expected at very large $R$, when the 'vapour' is very rarefied, however this limit is not observed in our calculations due to the large magnitude of $\phi(b)$.

The small rod lengths $b=0$ and $b=0.01$ give rise to more pronounced inhomogeneous density profiles than the larger rods $b=0.1$, as is illustrated in Figure 6, and therefore show a bigger difference between the homogeneous and inhomogeneous profile MFDFT free energies.

It can be seen from these plots that the MFDFT free energies always lie above the exact free energy. For homogeneous density profiles neither LDA nor RPA approximations are made in the functional (33), and it is an exact representation of equation (9). In this case, the Bogoliubov inequality ensures that the MFDFT free energy lies above the exact free energy. The large discrepancy between the exact free energy and the MFDFT free energy for homogeneous profiles is due to the inaccuracy of the perturbation expansion (8). The mean field $V(r)$, which in the homogeneous case is just a constant potential, is supposed to emulate the effect of attractive interactions $\phi\left(r-r^{\prime}\right)$ in order to make $\left\langle H_{1}^{2} / 2\right\rangle_{0}$ and higher order moments of $H_{1}$ as small as possible. This does not seem to be accurate for the cases considered.

As we are in a regime in which the interaction strength $\alpha$ is large, and the range $1 / \lambda$ is finite, an inhomogeneous mean field and therefore an inhomogeneous density profile is expected to do better. However in this case there will also be an implicit error due to the LDA and RPA in the functional. In order to quantify the error due to the LDA, a numerical investigation has been carried 
out in which the free energy of two hard rod particles in an external harmonic potential was calculated exactly and compared with the free energy calculated using the LDA approximation (13). It was found that the LDA performs extremely well up to very high ratios of potential well curvature to rod length, indicating that the LDA contributes negligibly to the error, at least in the parameter ranges for which inhomogeneous profiles were obtained in this study. Therefore for the case of inhomogeneous profiles, the difference between the MFDFT results and the exact free energy can be attributed again to a breakdown of the perturbation expansion leading to non-negligible higher order terms, as well as an error due to the RPA approximation in the evaluation of $\left\langle H_{1}\right\rangle_{0}$.

The large discrepancy between the exact and MFDFT free energy is due to the unsuitability of a mean field treatment for a system of low dimensionality, consisting of very few strongly interacting particles. For such a system large fluctuations or deviations from mean field behaviour are expected.

The correction $\Delta F$ for translational motion of the system centre of mass is significant for all three rod sizes. The profiles become narrower for smaller $b$, indicating that the mean field potential $V(r)$ is narrower, leading to stronger confinement of the centre of mass. This gives rise to a larger free energy correction factor for narrower profiles. It should be noted that once the translational correction $\Delta F$ is made, the Bogoliubov inequality is no longer valid regardless of the LDA and RPA approximations, since the confinement of the system centre of mass, the effect we are attempting to correct, is an inherent part of the $H_{0}$ reference system which includes an external field.

A numerical study on three profiles for $b=0.1,0.01,0$ with $R=5.0$ was carried out to evaluate the first order density profile $\rho_{1}(r)$ given by equation (23). This revealed that $\rho_{1}(r)$ is similar to $\rho(r)$ but is slightly flatter, as would be expected. This is illustrated in Figure 7 for the case of $b=0.1$. The free energy calculated from the resulting first order profiles did not vary significantly from the original zeroth order free energy obtained using the $\rho(r)$ profiles.

\section{CONCLUSIONS}

We have investigated how well a mean field density functional theory performs in a very simple case of two attractive hard rods on a ring. Mean field theories are expected to be less successful in treating small systems due to the importance of fluctuations: they are better suited as a description of large systems. Reguerra and Reiss point out ${ }^{31}$ that the neglect of certain fluctuations in MFDFT may be a blessing in disguise for application to nucleation theory. This is because it allows MFDFT to capture stationary states of a system that do not correspond to equilibrium states, as for example the formation of critical molecular clusters in a condensing vapour. In this study we focused on how well MFDFT can estimate the equilibrium free energy of a closed system, so this neglect of fluctuations represents a shortcoming highlighted in the difference between the MFDFT and exact free energies.

Our primary concern in this work is to see how the standard MFDFT functional should be modified to cope with finite size effects. Two modifications involve changes to the random phase approximation, used to estimate the interaction free energy. The first is the need to ensure normalisation of the two-particle distribution function when it is replaced by a product of single distribution functions. The second correction is to prevent the inclusion of forbidden configurations with overlapping hard rods. Failure to remove these configurations leads to a substantial overestimation of the cohesive energy.

The final effect concerns the loss of translational symmetry due to the introduction of a mean field potential. This means that the free energy associated with the motion of the system centre of mass is not correctly treated, and for small systems, the error can be substantial. This loss of symmetry remains even if higher order terms are included in the evaluation of the density profile. The problem is resolved by the replacement of the effective Hamiltonian controlling the motion of the centre of mass. The correction to the free energy can be evaluated directly using the density distribution in the reference hard sphere system, and is added to the MFDFT free energy once an optimal density profile (or equivalently mean field potential $V(r)$ ) has been found.

The modifications we have employed lead to substantial changes in the MFDFT free energy. We have compared these free energies with exact values, which are easy to compute for such a small system. We have highlighted the modification to the translational contribution to the free energy. Our calculations show that the correction is greatest when the optimal density profile departs most strongly from homogeneity.

We expect these various factors to be important in calculations of the free energy of small molecular clusters frequently made in nucleation studies using mean field density functional theory. Further studies along these lines are planned.

1 D. Kashchiev, Nucleation: Basic theory with Applications (Butterworth Heinemann, 2000).

2 I. J. Ford, Phys. Rev. E 56, 5615 (1997).

3 J. K. Lee, J. A. Barker, and F. F. Abraham, J. Chem. Phys. 58, 3166 (1973).

${ }^{4}$ P. R. ten Wolde and D. Frenkel, J. Chem. Phys. 109, 9901 (1998).

5 K. Yasuoka and M. Matsumoto, J. Chem. Phys. 109, 8451 (1998).

${ }^{6}$ K. Yasuoka and M. Matsumoto, J. Chem. Phys. 109, 8463 (1998). 
7 P. R. ten Wolde, M. J. Ruiz-Montero, and D. Frenkel, J. Chem. Phys. 109, 9901 (1998).

8 K. J. Oh and X. C. Zeng, J. Chem. Phys. 110, 4471 (1999).

9 H. Vehkamäki and I. J. Ford, J. Chem. Phys. 112, 4193 (2000).

10 P. Schaaf, B. Senger, J. C. Voegel, R. K. Bowles, and H. Reiss, J. Chem. Phys. 114, 8091 (2001).

11 S. Yoo, K. J. Oh, and X. C. Zeng, J. Chem. Phys. 115, 8518 (2001).

12 D. J. Lee, M. M. T. da Gama, and K. E. Gubbins, J. Chem. Phys. 85, 490 (1986).

13 D. W. Oxtoby and R. Evans, J. Chem. Phys. 89, 7521 (1988).

14 V. Talanquer and D. W. Oxtoby, J. Chem. Phys. 100, 5190 (1994).

15 A. Laaksonen, V. Talanquer, and D. W. Oxtoby, Ann. Rev. Phys. Chem. 46, 489 (1995).

16 J. C. Barrett, J. Chem. Phys. 107, 7989 (1997).

17 J. C. Barrett, J. Chem. Phys. 111, 5938 (1999).

18 A. Dillmann and G. E. A. Meier, J. Chem. Phys. 94, 3872 (1991).

19 V. I. Kalikmanov and M. E. H. van Dongen, J. Chem. Phys. 103, 4250 (1995).

${ }^{20}$ L. Gránásy, J. Chem. Phys. 104, 5188 (1996).

21 M. Volmer and A. Weber, Z. Phys. Chem. 119, 277 (1926).

22 D. Reguera, R. K. Bowles, Y. Djikaev, and H. Reiss, J. Chem. Phys. 118, 340 (2003).

23 A. G. et. al., Phys. Rev. Lett. 79, 2466 (1997).

24 J. J. Binney, N. J. Dowrick, A. J. Fisher, and M. E. J. Newman, Theory of Critical Phenomena- an introduction into the renormalization group (Oxford University Press, 1992).

25 J. K. Percus, J. Chem. Phys. 75, 1316 (1981).

26 J. K. Percus, J. Stat. Phys. 28, 67 (1982).

27 V. I. Kalikmanov, Statistical Physics of Fluids - Basic concepts and applications (Springer, 2001).

28 S. A. Harris and I. J. Ford, J. Chem. Phys 118, 9216 (2003).

29 H. Reiss, J. L. Katz, and E. R. Cohen, J. Chem. Phys. 48, 5553 (1968).

30 F. M. Kuni and A. I. Rusanov, Physics Letters 29 A, 337 (1969).

31 D. Reguerra and H. Reiss, J. Chem. Phys. 120, 2558 (2004). 


\section{Figures}

Figure 1. Comparison between the free energies of two non-interacting point particles and two interacting hard rods, as a function of ring circumference $R$. The difference between the two defines the excess free energy examined in Figures 3-5 for various cases.

Figure 2. Percentage error of MFDFT free energy for a homogeneous profile as a function of the potential well depth at contact $\alpha_{0}$, and interaction range parameter $\lambda$. For this plot $b=0.1$ and $R=5.0$.

Figure 3. Excess free energy calculated exactly (solid line), and in three different forms of MFDFT. The dashed line results from inserting a homogeneous density profile $\rho=2 / R$ into equation (33), the free energy functional incorporating the modifications to the random phase approximation. The squares show the same free energy evaluated using the optimal inhomogeneous density profile. This free energy lacks the translational correction $\Delta F$ described in equation (45), and the substantial change that this introduces is shown by the further shift to the values shown by circles. These calculations are performed for rods with length $b=0.1$, in dimensionless units, and for a specified attractive potential. The variation with $R$, the length of the ring, is shown. For most values of $R$, inhomogeneous density profiles are favoured, but homogeneous profiles become more favourable for smaller $R$. The translational correction greatly improves the agreement between the MFDFT and exact free energies.

Figure 4. As in Figure 3, but for rods with length $b=0.01$.

Figure 5. As in Figure 3, but for point-like rods with length $b=0$.

Figure 6. The optimal inhomogeneous density profiles for the three cases described in Figures 3-5 for $R=5$. Note that the smaller rods are described by a more peaked profile, and consequently a narrower mean field potential.

Figure 7. The zeroth (solid line) and first order density (dotted line) density profiles for the $b=0.1$ case, and the difference between them (dash-dotted line), according to the perturbative expressions given in section III B. 


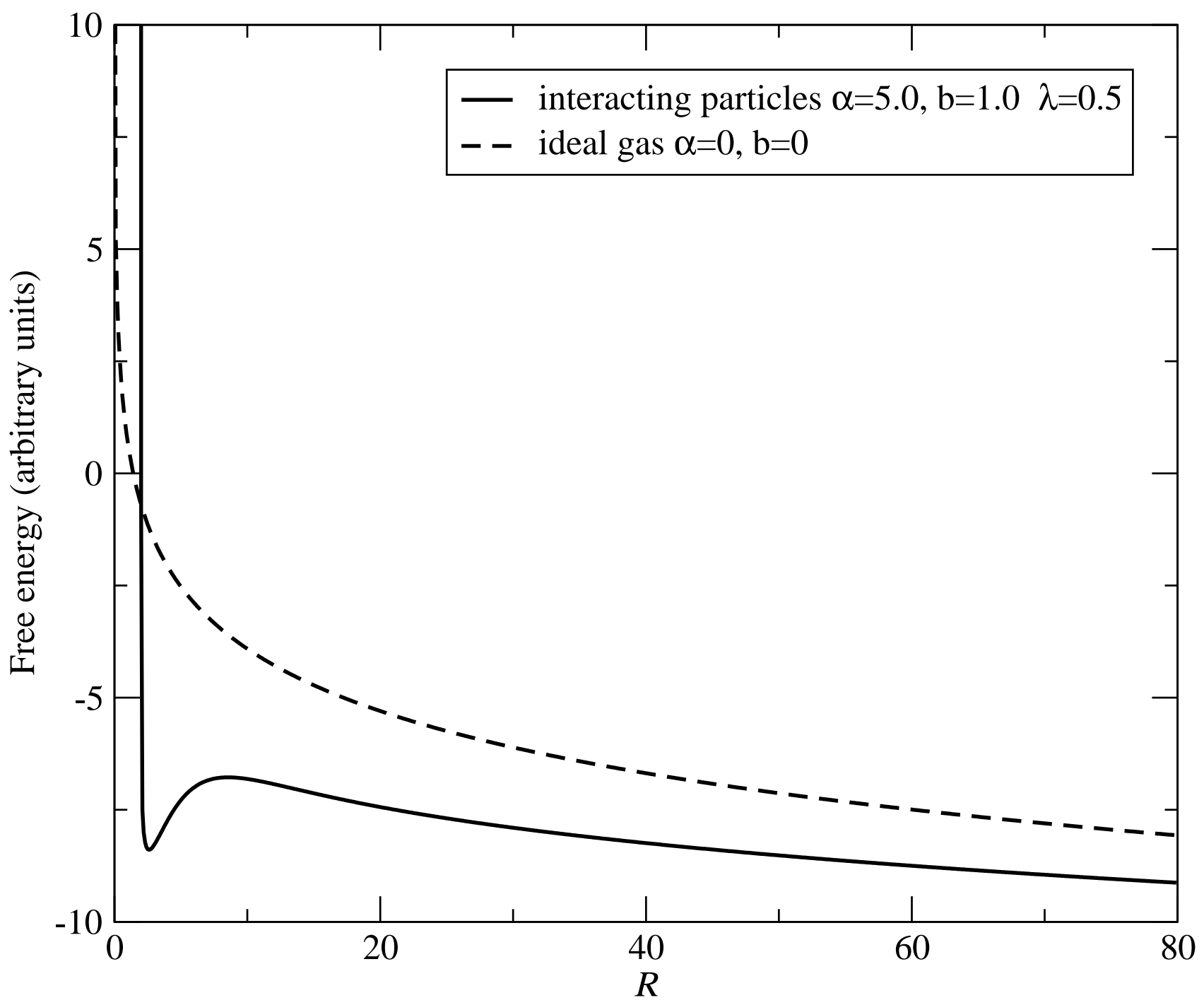

Figure 1: 


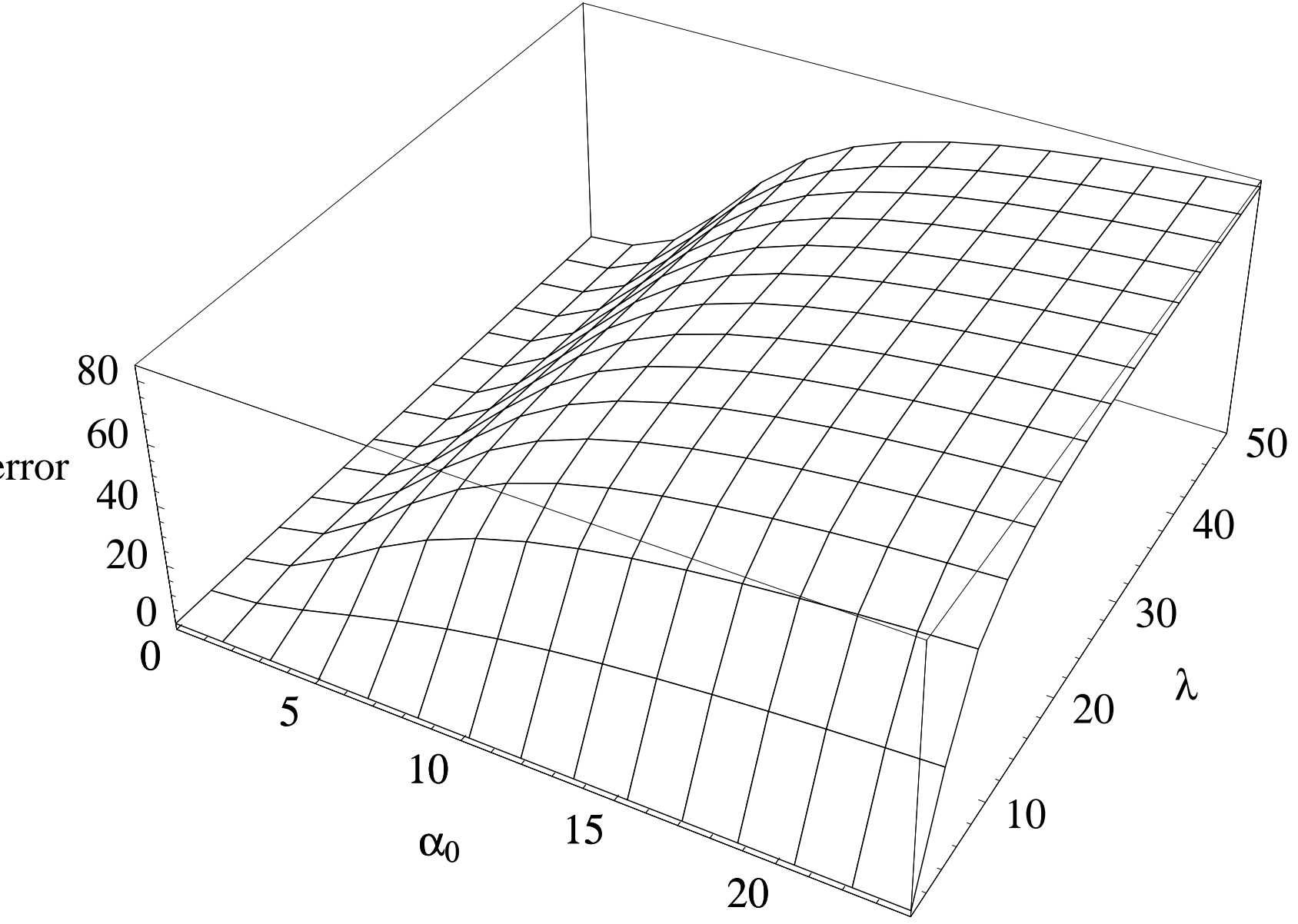

Figure 2: 


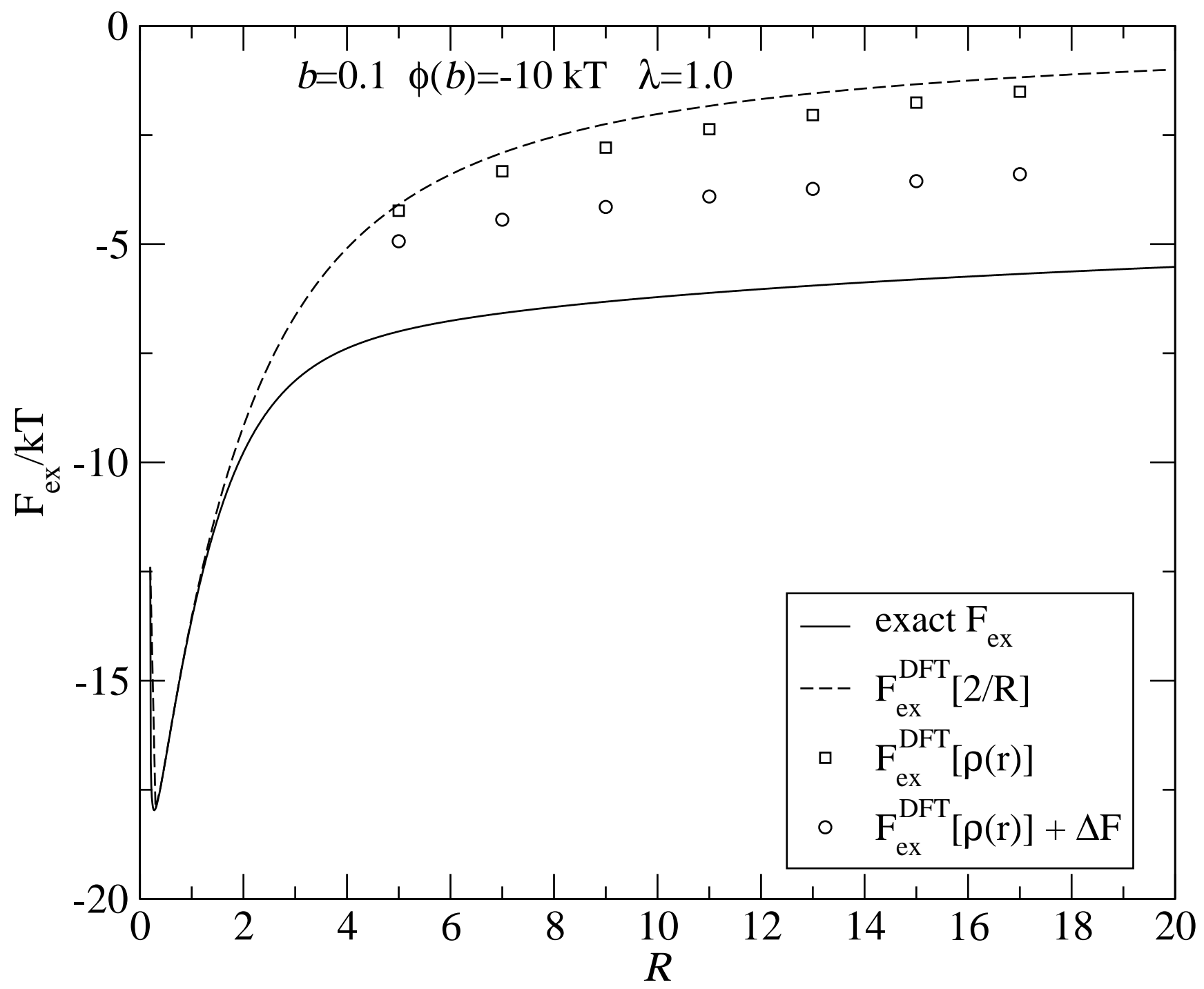

Figure 3: 


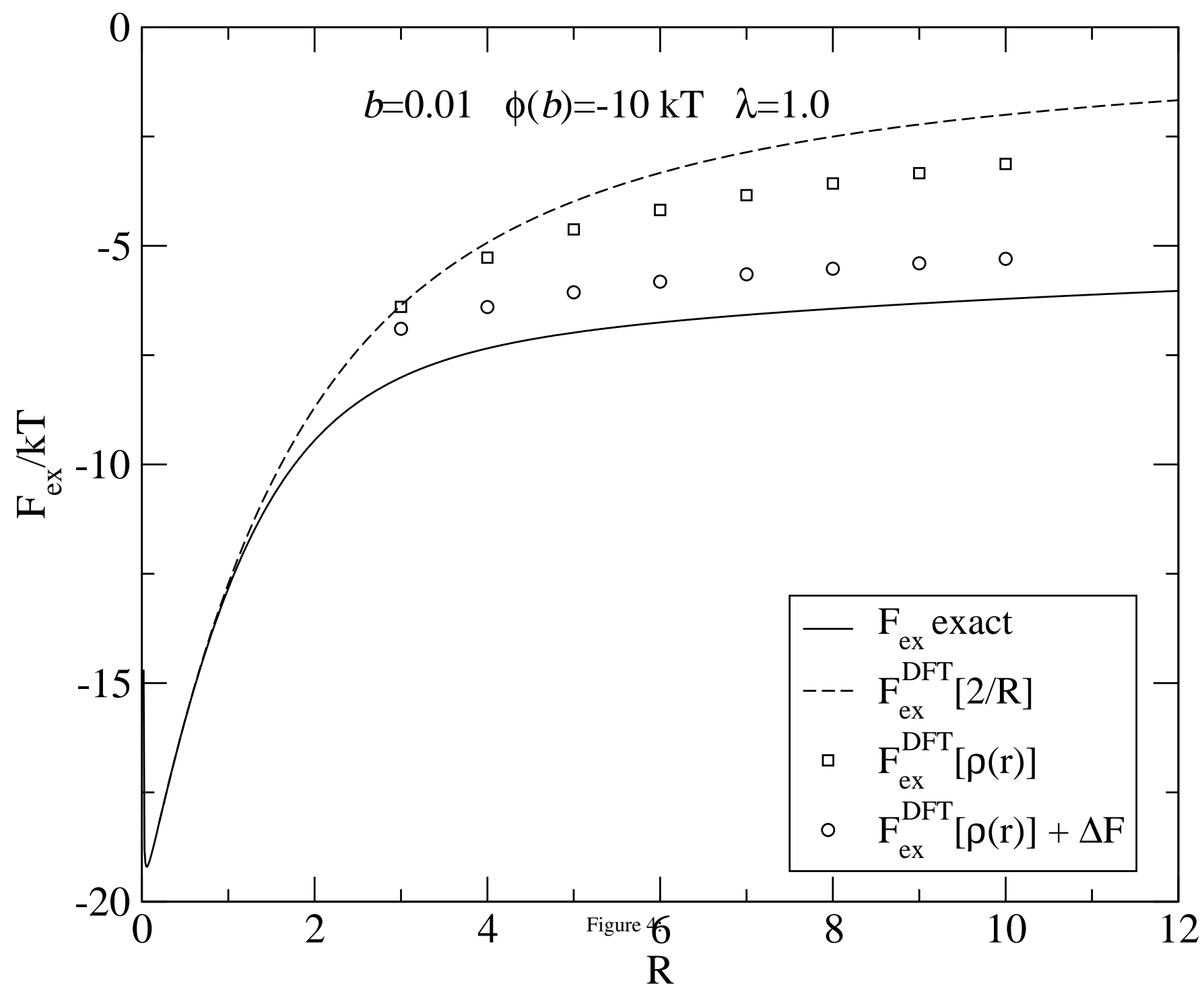




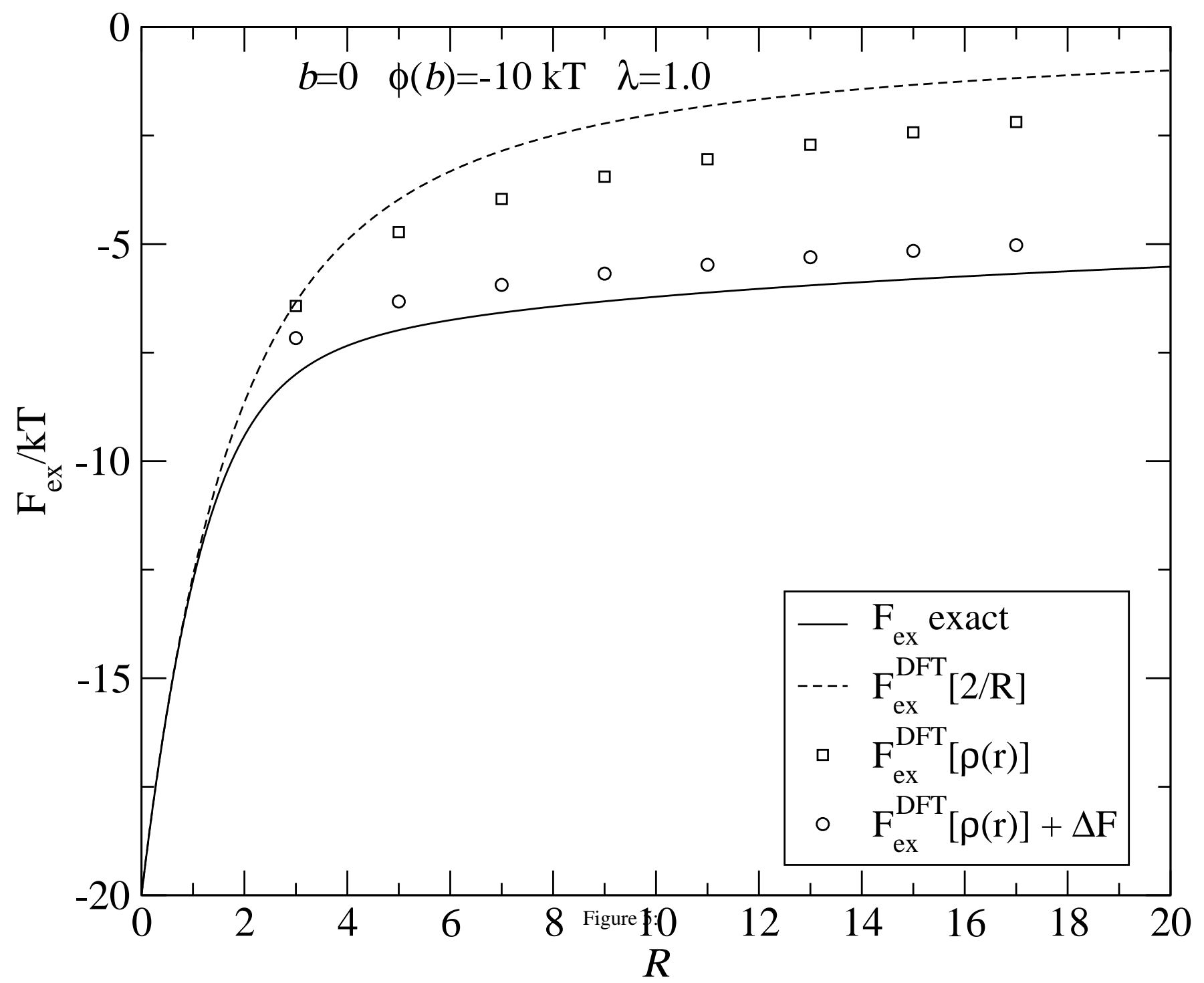




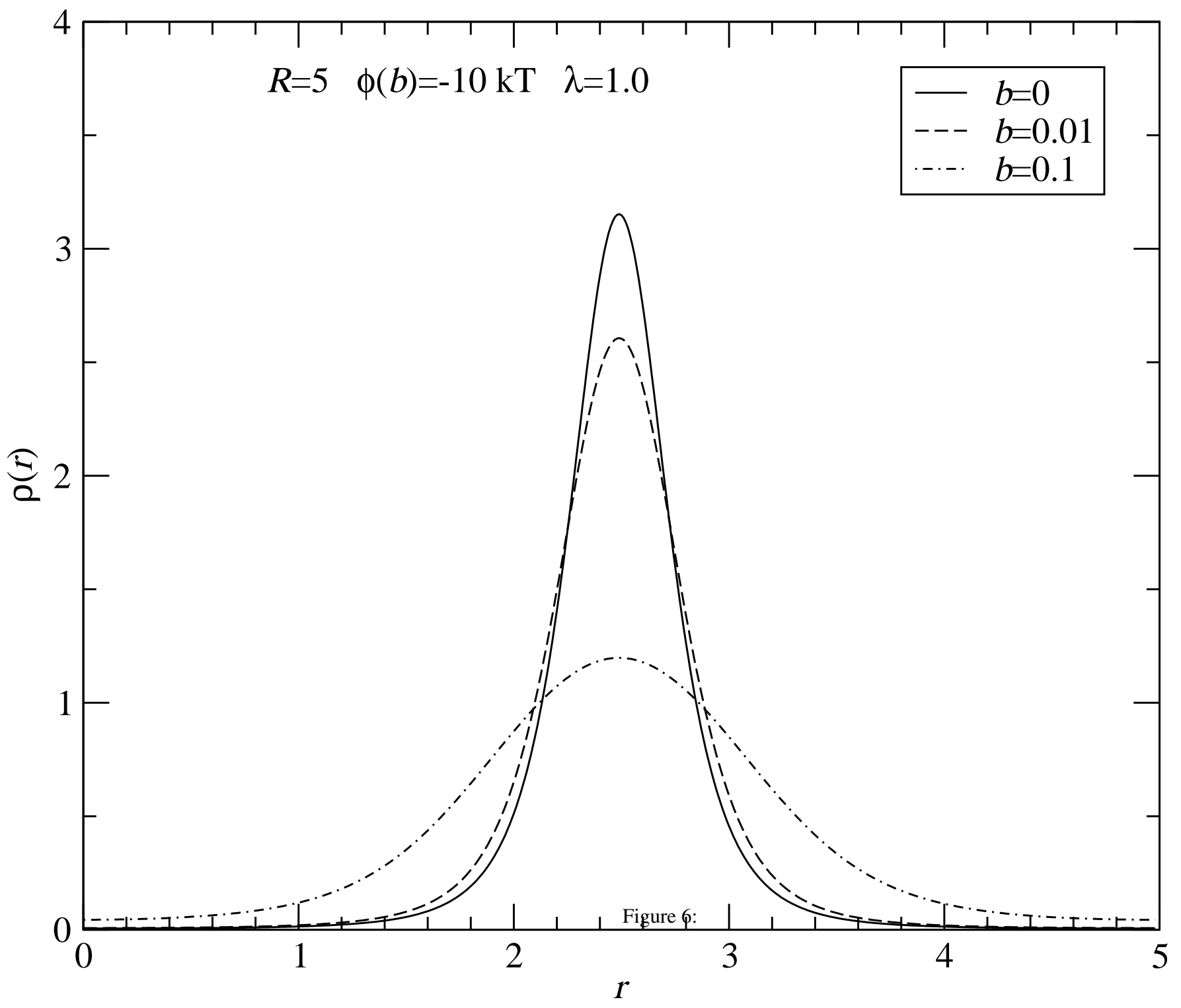




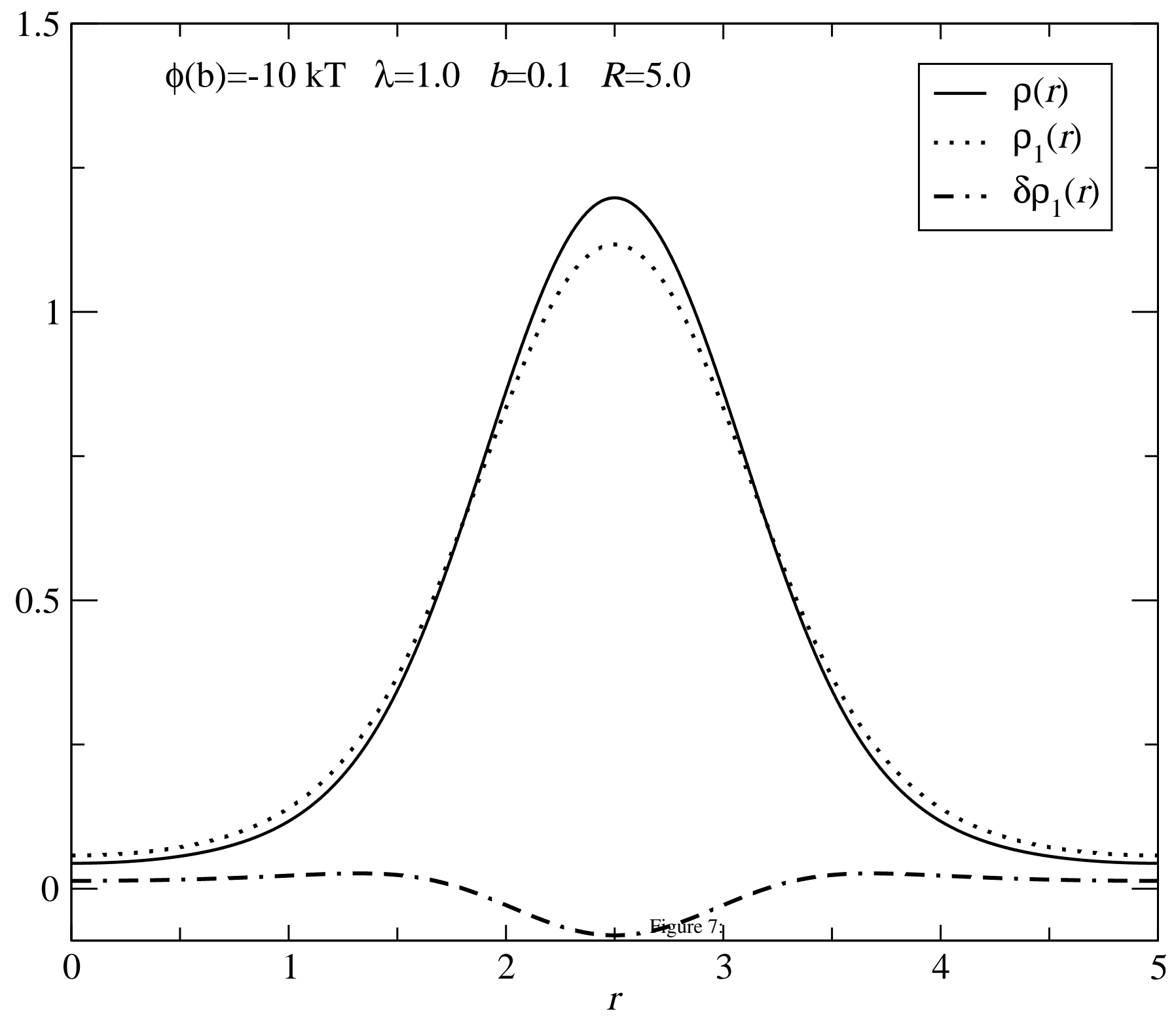

